Mathématiques et sciences humaines
Mathematics and social sciences

183 | Automne 2008

Hommage à Georges-Th. Guilbaud

\title{
Biographie de G.-Th. Guilbaud. Repères chronologiques
}

G.-Th. Guilbaud biography

\section{(2) OpenEdition \\ Journals}

Édition électronique

URL : http://journals.openedition.org/msh/10743

DOI : $10.4000 / \mathrm{msh} .10743$

ISSN : 1950-6821

Éditeur

Centre d'analyse et de mathématique sociales de l'EHESS

Édition imprimée

Date de publication : 14 décembre 2008

Pagination : 9-15

ISSN : 0987-6936

Référence électronique

«Biographie de G.-Th. Guilbaud. Repères chronologiques », Mathématiques et sciences humaines [En ligne], 183 | Automne 2008, mis en ligne le 15 décembre 2008, consulté le 23 juillet 2020. URL : http:// journals.openedition.org/msh/10743; DOI : https://doi.org/10.4000/msh.10743 


\title{
BIOGRAPHIE DE G.-Th. GUILBAUD
}

\author{
REPÈRES CHRONOLOGIQUES
}

[Nous remercions Marie-Anne et Pierre Guilbaud, ainsi que Bernard Bru, Michèle Frey et Georges Morlat, de nous avoir fourni de précieuses indications (la rédaction)].

- 26 décembre 1912 : Naissance à Brest (Finistère)

- Son père, Théodule Guilbaud y exerce la profession de comptable, et s'y est marié en 1911.

- La famille Guilbaud est originaire de La Bruffière, en Vendée nantaise ; elle est divisée, comme c'est traditionnel dans cette région, en «bleus » et «blancs »; ces Guilbaud-là (le nom est très fréquent localement) sont «bleus ».

- Théodule Guilbaud est cousin, issu de germain, du célèbre commandant Guilbaud, de l'aéro-navale, qui disparut en 1928 alors qu'il essayait, en avion, avec l'explorateur norvégien R. Amundsen, de retrouver l'expédition italienne de Nobile, qui avait disparu dans l'Arctique.

- Sa mère, Fernande Thépot, appartient à une famille brestoise depuis plusieurs générations. Fille d'un artisan ébéniste, elle a une sœur et trois frères dont deux, Georges et Louis, anciens élèves de l'École Navale, sont ingénieurs de la marine et termineront leur carrière comme ingénieurs en chef, avec rang d'amiral. Ils participent aux activités du «Sillon» de Marc Sangnier, implanté à Brest dès 1900.

- De ce mariage naîtront trois autres enfants: Paul, mort en bas âge en 1914, Jacqueline en 1922 et Pierre en 1925.

- 1914-1918 : Théodule Guilbaud est mobilisé dans le service des explosifs et de la pyrotechnie de l'armée. C'est là que se confirme son intérêt pour la chimie (il pratiquait la photographie en amateur). Il y devient ami de Daniel Berthelot, neveu du grand Marcellin, et lui-même chimiste. Théodule est réformé à la suite d'un accident dans les manipulations chimiques.

- 1918-1923 : Son ami et lui inventent un nouveau type de carburateur pour les moteurs d'automobiles. Pour en exploiter les brevets, ils créent une société, BERGUIL (Berthelot-Guilbaud) dont le siège est à Meudon (Hauts-de-Seine, alors Seine et Oise).

- Théodule Guilbaud et sa famille s'installent à Paris.

- 1924: La société BERGUIL est achetée par le grand fabricant lyonnais de carburateurs, la S.A. ZENITH.

- La famille s'installe à Lyon, où Georges Th. est élève au lycée du Parc.

- 1928 : La filiale de ZENITH à Turin, qui est fournisseur pour les automobiles FIAT, connaît des difficultés. 
- Théodule Guilbaud est envoyé, comme conseiller technique, à ZENITH-Turin.

- La famille déménage à Turin, mais Georges Th. n'y fera que de brefs séjours : il est interne au Lycée du Parc à Lyon jusqu'à l'année 1930-1931 où il y est élève en classe de mathématiques supérieures.

- 1928-1931 : Ces années d'internat à Lyon vont jouer un rôle décisif dans la formation de sa personnalité.

- Outre l'acquisition définitive de son amour pour les mathématiques, c'est pendant cette période que prennent corps deux autres orientations fondamentales de son existence : l'amour de la montagne et celui des monastères. Il dira plus tard : ses «trois $\mathrm{M} »$.

- Amour de la montagne : la famille passe ses vacances à Lanslebourg, près du Mont-Cenis, à mi-chemin entre Lyon et Turin. C'est dans cette haute vallée de la Maurienne, pour laquelle son père avait eu un «coup de foudre » lors de son premier voyage de Lyon à Turin, que George Th. aimera revenir chaque année. Il y passera des vacances surtout à Lanslevillars des années 1950 aux années 1990.

- Amour des monastères : en 1930, il fait une première retraite dans une abbaye cistercienne, celle des Dombes, dans l'Ain, où le R.P. Couturier, qui jouera un grand rôle dans sa vie, a créé un groupe œcuménique.

Il s'ensuivra une fréquentation permanente des cisterciens et de nombreux séjours dans leurs abbayes : Orval (en Belgique), Citeaux, bien sûr, Port-du-Salut, près de Laval (cf. l'article de B. Bru, p. 55).

C'est aussi au cours de ces années lyonnaises que, par l'intermédiaire d'un prêtre qui œuvre auprès d'ouvriers émigrés nord-africains, Georges Th. rencontre l'Islam, pour lequel son intérêt perdurera; plus tard, il apprendra l'Arabe et étudiera le Coran.

Georges Th. sera d'ailleurs toujours passionné par les langues (et, partant, la linguistique). C'est ainsi qu'il pratiquera l'Hébreu, pour lire la Bible, le Russe, le Portugais et se mettra même, dès sa jeunesse, au Japonais et au Chinois. Bien entendu, il connaissait parfaitement l'Anglais, et parlait couramment l'Italien ; il avait dans ses études secondaires, connu la classique trilogie des «bons élèves »: Latin-Grec-Allemand.

- 1931-1932 : Interne, en classe de mathématiques spéciales, au lycée Saint-Louis de Paris.

- Il passe le concours d'entrée à l'École normale supérieure (où il est admis) et, à la demande de son père, celui de l'École Polytechnique, où il est reçu $1^{\mathrm{er}}$.

- Il opte pour l'ENS, par vocation. Son père lui a laissé le choix.

- 1932-1935 : Élève à l'ENS, il passe la licence de mathématiques, qu'il complète par le certificat de calcul des probabilités et de statistique de Georges Darmois. C'est son premier contact avec ces deux disciplines, qui occuperont une si grande place dans sa vie professionnelle et scientifique. Deux décennies plus tard, Guilbaud collaborera avec Darmois dans le cadre de l'Institut de statistique de l'Université de Paris.

- Simultanément, il suit au Collège de France le premier cours d'Etienne Gilson en philosophie médiévale, qui porte sur la théologie mystique de Saint-Bernard.

- Il fait partie du groupe des «talas » de l'ENS ; il y fait la connaissance de Paul Vignaux, ancien élève et de dix ans son aîné, spécialiste de philosophie médiévale, et futur fondateur du mouvement «Reconstruction » de la CFTC, à l'origine de la transformation de celle-ci en CFDT. 
- Plus tard, Georges Th. sera membre de la Paroisse universitaire et aura des rapports suivis avec «Économie et Humanisme».

- Au cours de cette même période, qui fut celle de l'arrivée de Hitler au pouvoir en Allemagne (30 janvier 1933) et de l'émeute du 6 février 1934 à Paris, il milite dans les mouvements républicains et antifascistes.

- Sa connaissance de l'Italie et de sa langue lui permettent d'ailleurs des contacts avec des étudiants italiens réfugiés à Paris.

- À l'ENS, Georges Th. a pour condisciple Pierre Costabel, également mathématicien, qui deviendra oratorien et historien des mathématiques des $\mathrm{XVII}{ }^{\mathrm{e}}$ et XVIII ${ }^{\mathrm{e}}$ siècles. Les liens d'amitié et de collaboration entre Guilbaud et Costabel dureront jusqu'à la mort de ce dernier, en 1989.

- 1935 : Reçu à l'agrégation de mathématiques.

- 1935-1936 : Service militaire à Metz, dans l'artillerie antiaérienne. Il en sort officier de réserve.

- $1^{\text {er }}$ juillet 1936 : Mort de son père, Théodule Guilbaud. À partir de cette date, et bien que rien ne l'y obligeât, il prend sa famille à sa charge. Jusqu'à l'après-guerre sa mère, sa sœur et son frère le suivront dans ses diverses affectations.

- 1936-1937 : Professeur au lycée de Nevers.

- 1937-1939 : Professeur de mathématiques spéciales au lycée de Metz. Il y fait, en 1938, la connaissance du futur psycho-sociologue Roger Daval, que nous retrouverons dans la suite, nommé professeur de philosophie ; Roger Daval devient et restera un ami de la famille Guilbaud.

Pendant cette période messine, Georges Th. fait des séjours au monastère cistercien d'Orval (en Belgique); il aide les moines à reconstituer leur bibliothèque qui était tombée en déshérence.

- 1938 : Il est rappelé sous les drapeaux lors de la crise qui aboutit aux accords de Munich (fin septembre).

- 1939-1940 : Mobilisé, comme lieutenant d'artillerie antiaérienne, il est sur la ligne Maginot pendant la plus grande partie de la guerre.

Après la débâcle de juin 1940, il est démobilisé à Moissac (Tarn-et-Garonne) au cours de l'été.

La croix de guerre lui est attribuée.

- 1940-1941 : Nommé (en mathématiques spéciales) au lycée de Brest (Metz annexée par le III ${ }^{\mathrm{e}}$ Reich, était désormais inaccessible).

En raison des bombardements de l'aviation anglaise, la ville est évacuée et le lycée est transféré à Quimper à Pâques 1941.

- Octobre 1941 : Nommé au lycée de Dijon, toujours comme professeur de taupe ; il y restera jusqu'en 1947. G.G. Granger y arrive en 1943: la redécouverte et la réhabilitation de la mathématique sociale de Condorcet va résulter de leur rencontre.

De 1942 à 1947 Georges Th. est chargé d'un cours de philosophie des sciences à la Faculté des lettres et sciences humaines de Dijon. Ce n'est qu'un début !

- 1941-1944 : Georges Th. a toujours été très discret sur son action de résistance. Ce qui est certain, c'est d'abord qu'il organisa à Dijon la diffusion du Témoignage chrétien clandestin des années d'occupation. C'est aussi qu'il eut des contacts avec des réseaux de résistants, probablement comme agent de liaison entre eux, ou avec des émissaires venus secrètement d'Angleterre ou d'Algérie. 
- 1945 : Publication de son premier livre, en collaboration avec Roger Daval Le raisonnement mathématique, Paris, Presses Universitaires de France, 152 p.

- 1946 : Son mariage avec Solange Dupin, professeur agrégé de lettres. De cette union naissent deux enfants, Marie-Anne en octobre 1947 et Jean en juin 1949.

- 1947 : Il entre à l'Institut de Science Économique Appliquée (ISEA) dirigé par François Perroux, comme chargé de recherche. Il y restera jusqu'en 1955 et en deviendra le directeur adjoint en 1951.

Il s'installe à Paris, puis, en 1949, à Saint-Germain-en-Laye qu'il ne quittera plus.

- 1947-1955 : Pendant qu'il est à l'ISEA, Georges Th. a une activité intense.

- Ses articles et les livres qu'il publie (cf. sa bibliographie, p. 17) introduisent en France le nouveau calcul économique, la théorie des jeux, celle des décisions collectives, la recherche opérationnelle, la programmation linéaire.

Plusieurs de ces textes sont traduits et publiés dans des revues scientifiques étrangères. Certains, parus dans la revue Economie appliquée seront ultérieurement réunis en volume.

- Il enseigne simultanément à l'École d'application de l'INSEE (la future ENSAE) à partir de 1948, à «Sciences Po » et à l'Institut Statistique de l'Université de Paris (ISUP) à partir de 1949, à l'Institut de préparation scientifique aux études économiques de l'université de Paris (1951 et sq).

- C'est l'époque où il noue des liens de collaboration avec de grandes entreprises et avec les organismes publics de pilotage de l'économie (cf. interview réalisée par Denis Bayart, p. 35).

Il est notamment rapporteur à la $6^{\text {e }}$ section (comptabilité nationale) du Conseil supérieur de la comptabilité (1950 et sq.) et membre de la commission des comptes de la nation où il est nommé en 1952.

- 1950 : Élection au conseil de la Société de statistique de Paris.

C'est cette année que Georges Th. fait la connaissance de Jacques Lacan. Les deux hommes s'estiment mutuellement et échangeront souvent des idées lors de conversations privées (voir à ce sujet: Elisabeth Roudinesco, Histoire de la psychanalyse en France (la bataille de cent ans) 2 : 1925-1985, Paris, Le Seuil, 1986, p. 564-565).

- 1951 : «Fellow » de la Société internationale d'économétrie.

- 1953 (mai) : Création et direction du séminaire de recherche opérationnelle de l'ISUP qui jouera un rôle fondamental pour la diffusion de cette discipline nouvelle dans les entreprises françaises (on pourra voir à ce sujet, et pour tout ce qui concerne les débuts de la recherche opérationnelle en France : B. Roy, «Regard historique sur la place de la recherche opérationnelle et de l'aide à la décision en France », Mathématiques et Sciences humaines 175, 2006(3), p. 25-40).

C'est cette même année 1953 que le colloque international Econométrie du CNRS réunit à Paris pour la première fois depuis la guerre nombre de futurs « Prix Nobel » d'économie. Guilbaud en est l'un des principaux animateurs.

- 1955 : Élection à l'École Pratique des Hautes Études (EPHE), 6 e section, dans une direction d'études de Méthodes mathématiques des sciences sociales. Il y poursuivra toute sa carrière - sans préjudice de nombreuses activités annexes - jusqu'à sa retraite en 1981.

- 1956 : Création et direction au sein de l'ISUP du bureau universitaire de recherche opérationnelle (BURO), installé, jusqu'en 1958, 2 rue Chauchat, $9^{\mathrm{e}}$ arrondissement. 
Y sont affectés : Micheline Petruszewycz, Edouard Valette, Françoise Peyrot et Marc Barbut.

Tous les samedis matin, réunions de «brainstorming » mathématique auxquelles participent Jean Bouzitat, Maurice Girault, Germain Kreweras et Georges Morlat.

- 1956 (juin) : Création de la Société Française de Recherche Opérationnelle (SOFRO) dont Georges Th. est élu président, et de la Revue française de recherche opérationnelle.

- 1957 : Création des Cahiers du BURO.

Lauréat de l'Académie des sciences (Prix Montyon de statistique pour 1957) «pour ses travaux de calcul des probabilités, de théorie des jeux et leurs applications ».

- 1958 : Création, au sein de l'EPHE, $6^{\mathrm{e}}$ section, du Groupe de Mathématique Sociale et de Statistique (GMSS) qui s'installe 17 rue Richer $9^{\mathrm{e}}$ arrondissement, les «Folies Guilbaud» (les « Folies Bergères » sont presque en face).

Dans cet ancien hôtel des maréchaux de Napoléon $1^{\text {er }}$, cohabite et collabore avec le GMSS, l'Institut des Sciences Humaines Appliquées (ISHA) de l'Université de Paris, dirigé par Roger Daval, qui partage son bureau avec George Th. Guilbaud.

Les deux organismes sont réunis sous l'appellation non officielle mais usuelle de Centre Condorcet. Cela durera jusqu'au transfert (fin 1969) à la Maison des Sciences de l'Homme, 54 boulevard Raspail $6^{\mathrm{e}}$ arrondissement, du Centre de mathématique sociale.

Car le GMSS deviendra Centre de Mathématique Sociale et de Statistique (CMSS) en 1962, Centre de Mathématique Sociale (CMS), laboratoire associé au CNRS en 1967, et enfin Centre d'Analyse et de Mathématique Sociales (CAMS), unité mixte EHESS-CNRS en 1980.

C'est à cette époque (1958 ou 1959) que se noue une autre amitié marquante, celle avec Louis Frey (décédé en 1999), futur titulaire de la chaire de logique de l'Université de Provence, à Aix. Guilbaud dirigera la thèse de Louis Frey sur «l'analyse ordinale des évangiles synoptiques ». Louis et Michèle Frey feront plusieurs séjours d'été à Lanslevillard, chez les Guilbaud.

- 1959-1963 : Professeur à la Faculté de droit et sciences économiques de Paris, où il définit et organise l'enseignement de mathématiques devenu obligatoire en licence de sciences économiques.

- 1960 : Organisateur du colloque international du CNRS sur La décision. Il dirige la publication des actes (éditions du CNRS, 1961).

- 1962 (octobre) : avec Marc Barbut, création de la revue Mathématiques et Sciences humaines.

La décision en a été prise à l'issue d'un colloque tenu dans les locaux du Laboratoire d'anthropologie sociale, au sous-sol du Palais de Chaillot, et réunissant plusieurs dizaines d'enseignants des facultés des lettres et sciences humaines de province et de Paris.

- 1963 : Ernest Coumet (décédé en 2003), alors pensionnaire à la Fondation Thiers, épistémologue et historien des mathématiques, commence à fréquenter le Centre Condorcet. Il aura pour Guilbaud une admiration et une fidélité qui ne se démentiront pas.

- 1966 (et sq.) : Avec Pierre Rosenstiehl, il organise l'enseignement de mathématiques pour les études de sciences humaines et sociales à la Faculté des lettre et sciences humaines de Paris-Nanterre. 
- 1967 : Membre de la commission de réforme de l'enseignement des mathématiques présidée par André Lichnerowicz.

Organisation et direction du colloque international du CNRS $n^{\circ} 171$, La décision, agrégation et dynamique des ordres de préférence, Aix en Provence, 3-7 juillet 1967. Actes publiés aux éditions du CNRS, Paris, 1969.

- 1969 (décembre) : Le Centre de mathématique Sociale s'installe à la Maison des Sciences de l'Homme, 54 boulevard Raspail.

Au cours de la décennie 1960-1970 : les activités de G.-Th. Guilbaud peuvent être regroupées selon trois axes principaux.

- La praxéologie mathématique (un groupe de recherche, le GEMPPE, est créé, au sein $\mathrm{du}$ Centre de mathématique sociale, autour de Pierre Rosenstiehl) objet de collaborations avec des personnalités telles que Raymond Aron et Jacques Vernant (directeur du Centre d'études de politique étrangère). Mais aussi avec les institutions militaires, notamment, cours sur les mathématiques de la décision donnés aux Écoles de guerre.

- La combinatoire et l'algèbre et leurs applications qui engendrent des travaux en commun avec bien des équipes ou des chercheurs individuels en sciences humaines, parmi lesquels :

- le Laboratoire d'anthropologie sociale de Claude Levi-Strauss ;

- le Laboratoire de psychologie génétique de François Bresson et Pierre Gréco ;

- le compositeur Iannis Xenakis, dont l'équipe de mathématique musicale (EMAMU) est créée dans le cadre du Centre de mathématique sociale.

Le séminaire sur la modélisation mathématique dans les sciences de l'homme, à la Sorbonne, est un lieu privilégié d'échanges.

- La pédagogie des mathématiques, avec :

- l'organisation de stages de formation pour des enseignants et des chercheurs en sciences humaines ;

- la réalisation, en collaboration avec son frère, qui est cinéaste, de films d'animation pour l'initiation à la combinatoire, au calcul des probabilités et à la statistique ;

- l'animation et la co-direction, avec André Revuz (fondateur de l'Institut de Recherche sur l'Enseignement des Mathématiques - IREM de Paris) et Gilbert Walusinski (secrétaire général de l'Association des Professeurs de Mathématiques de l'Enseignement Public - APMEP), de la série d'émissions «Chantiers mathématiques » diffusées par la Télévision scolaire. Cette série sera suivie, dans les années 1970, par « Mathématiques pour tous ».

Sur ces deux derniers points, (cf. la filmographie de G.-Th. Guilbaud, p. 23).

À partir des « années 70 », ses intérêts sont principalement orientés vers :

- La statistique appliquée à la linguistique, en étroite collaboration avec le Laboratoire de lexicologie politique de la Fondation nationale des sciences politiques.

- Le langage de l'approximation, et les problèmes mathématiques qui s'y rapportent ; cela donnera notamment lieu à la publication, en 1985, des «Leçons d'à peu près » (cf. Bibliographie, p. 17). 
- L’histoire de la pensée mathématique, qui a toujours été présente dans son enseignement et ses travaux, mais qui l'amène à une participation très active au Séminaire d'histoire du calcul des probabilités et de la statistique (créé en 19821983, par E. Coumet, B. Bru et M. Barbut), où, notamment, il fera une dizaine de communications.

- 1981 : Admis à faire valoir ses droits à la retraite. Retraite qui sera très active jusqu'à la fin des « années $90 »$ au moins.

- 1986 : Colloque en l'honneur de G.-Th. Guilbaud, du 14 au 16 juillet à Urbino (Italie), autour des thèmes de l'approximation.

Les contributions sont publiées l'année suivante sous le titre «L'à peu près. Aspects anciens et modernes de l'approximation », aux éditions de l'EHESS.

Les dernières années de la vie de Georges Th. Guilbaud sont pour l'essentiel consacrées à sa femme, gravement malade et qui décède en septembre 2006. Mais il continue à lire, écrire, réfléchir et « faire des maths ».

- 23 mars 2008 (dimanche de Pâques) : Décès à Saint-Germain-en-Laye.

Septembre 1928

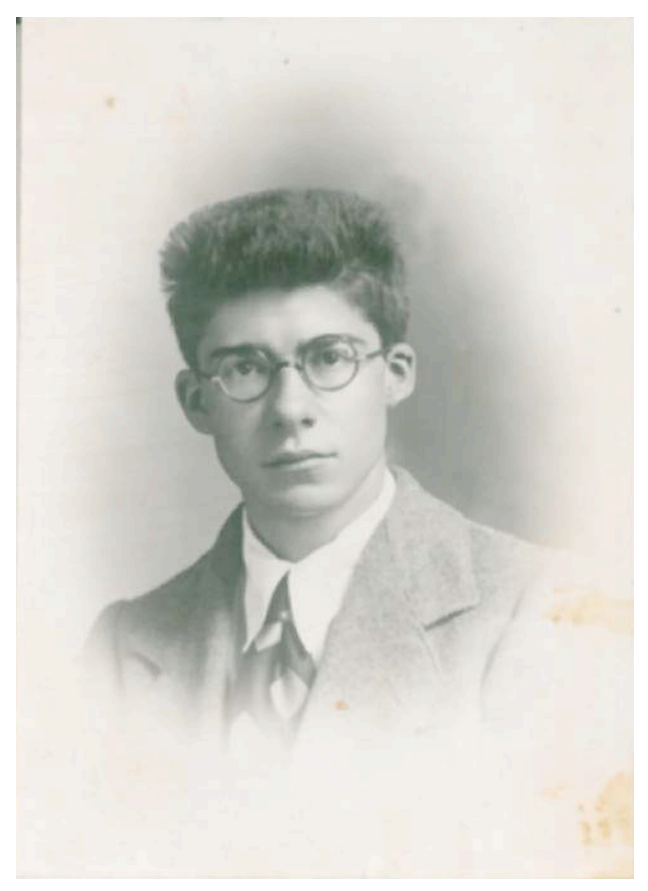

50 ans plus tard

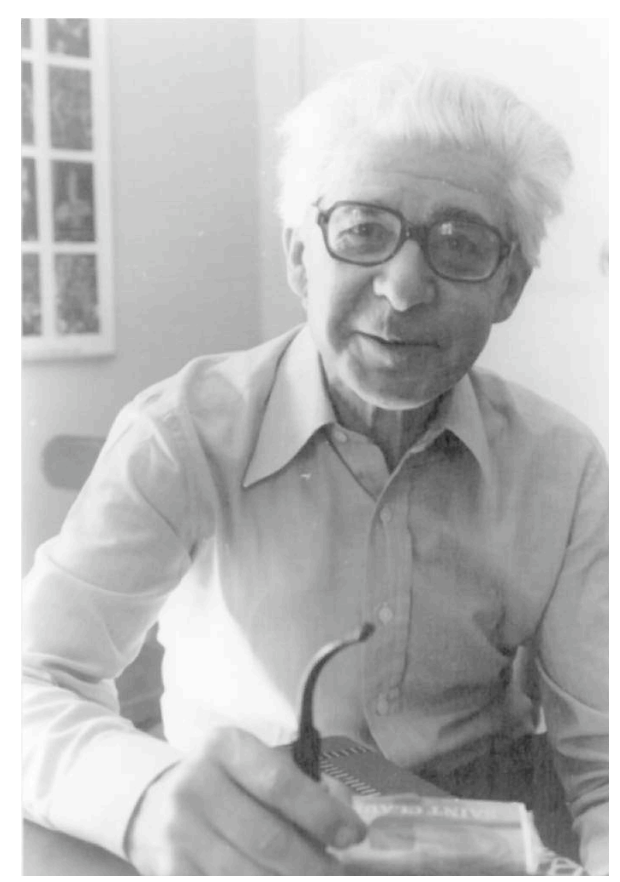

